\title{
Selectivity of a resonant cavity antenna
}

\author{
Viktor Bukharin ${ }^{*}$ and Nikolay Voytovich \\ FSAEIHE "South Ural State University (national research university)", Higher School of Electronics \\ and Computer Sciences, 454080 Chelyabinsk, Russian Federation
}

\begin{abstract}
The main selective characteristics of a Resonant Cavity Antenna, which is a radiating element of the antenna array of a glide path station, are presented. The results of rigorous electrodynamic modeling of a resonator antenna and experimental results of studies on antenna samples are presented
\end{abstract}

\section{Introduction}

A flat resonant cavity antenna (RA) serves as a radiating element of the antenna array of a ILS glide path station. The glide path beacon is part of a two-frequency radio beacon landing system for the UHF band ILS instrument landing system for aerodromes of civil aviation, including aerodromes with a high level of snow cover and difficult terrain.

The accuracy of ILS glide path depends on the stability of the parameters of the radiating elements of the antenna array when exposed to external environmental factors. Consequently, radiating elements with high spatio-temporal selective properties are of great practical interest.

RA has unique properties that can significantly reduce the impact on the characteristics of the radio system of unwanted radio waves, meteorological environmental factors and the underlying surface.

Resonator antennas have been known since the 50s of the 20th century [1]. All of them have one or several resonators located along one axis [1-11]. The longitudinal dimensions of the antennas exceed half the operating wavelength. In antennas there is a mode of mixed waves. This leads to a deterioration of the directional properties, polarization properties of the antenna and poor matching of the antenna with the feeder.

This paper presents the original aperture, two-cavity, single-mode RA, having a longitudinal size not exceeding half the working wavelength. RA works as a Fabry-Perot resonator. This paper is an extension of work originally presented in 29th International Crimean Conference "Microwave \& Telecommunication Technology" [12]. Illustrative material and information on the polarization properties of the antenna are added.

\section{Object of research}

The object of research is a flat resonant cavity antenna $[13,14]$. RA is an antenna array with a flat aperture, each radiator of which is in-phase excited by the main mode of the cavity resonator (Fig. 1). The antenna is built on the basis of two interconnected resonators:

* Corresponding author: bukharinva@susu.ru 
a cylindrical resonator with a flat partially transparent radiating wall and a coaxial slot resonator located into it. The cylindrical cavity of the antenna is excited by a coaxial slot radiator. The resonator antenna is made of aluminum alloy. It is a low volume cylindrical resonator with a partially transparent flat wall. The partially transparent wall is made of a flat plate on which round holes are made. It is the radiating aperture of the antenna.
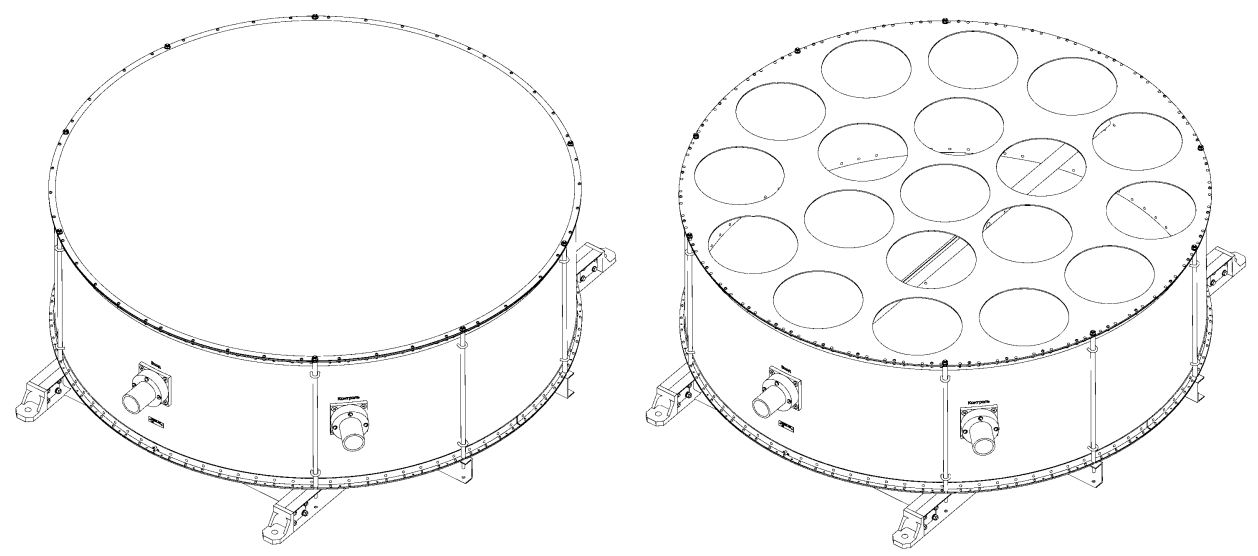

Fig. 1. Flat resonant cavity antenna.

The resonant cavity antenna does not have a complex diagram-forming circuit that distributes electromagnetic energy between radiators (holes). The role of the power divider between the radiating holes is performed by the volumetric cylindrical resonator.

\section{Research methods and basic characteristics of the resonant cavity antenna}

To study the characteristics of RA, numerical and field experiments were used. Experimental studies were conducted on a resonator antenna sample using a complex transmission and reflection coefficient meter "AGILENT E5071C ENA Network Analyzer". Theoretical studies were performed in a strict diffraction statement of the boundary-value problem by the space-time numerical method [15].

We consider a flat RA (Fig. 1), having the following geometric dimensions, expressed in wavelengths $\lambda$ : cylinder diameter is equal to $1.55 \lambda$; resonator length is equal to $0.497 \lambda$; wall thickness of partially transparent surface is equal to $0.0033 \lambda$; the diameter of the holes is equal to $0.272 \lambda$. The radiating holes are regularly located on two circles with radii $0.31 \lambda$ and $0.62 \lambda$. One hole is located in the center of the antenna aperture. The modulus of the reflection coefficient from a partially transparent surface is equal to 0.878 . The loaded Qfactor of a volumetric cylindrical resonator with a radiating aperture is equal to 29.46 .

\subsection{Frequency matching with a feeder of a resonant cavity antenna}

It has been theoretically and experimentally established that RA in a wide frequency band (several octaves) suppresses unwanted signals outside the working frequency band. Figure 2 shows the frequency dependence of the modulus of the reflection coefficient in a wide frequency band.

There is no consistent RA mode of operation in the frequency range of several octaves outside the operating frequency band. This circumstance is fundamental for solving the problems of noise immunity and electromagnetic compatibility of radio systems. Out-ofband signals cannot excite electromagnetic oscillations for which there is no self-consistent 


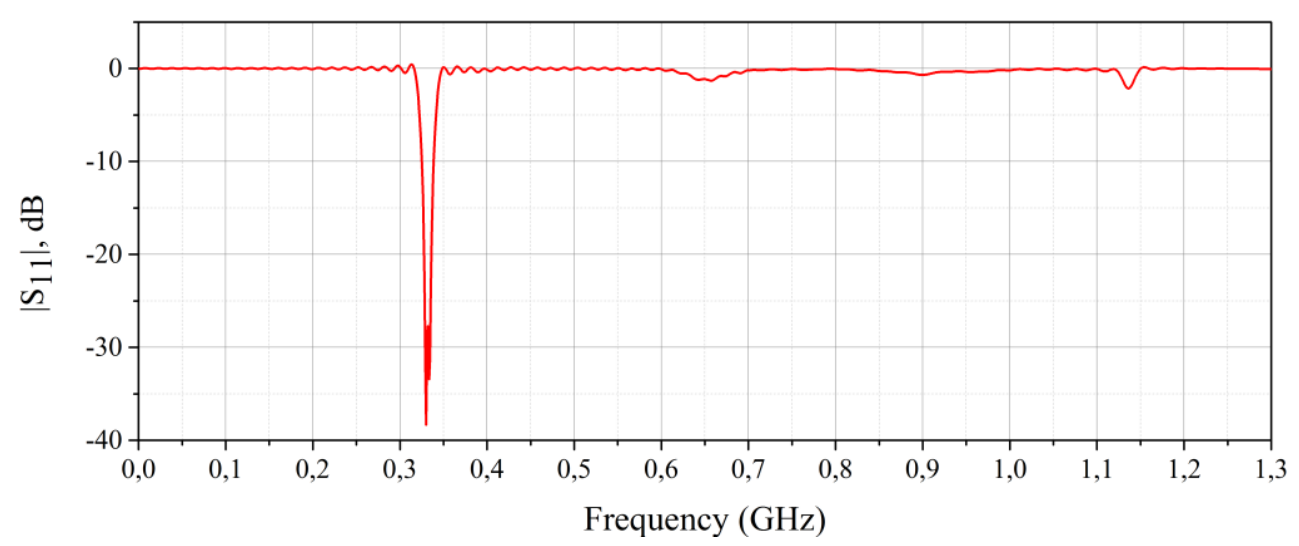

Fig. 2. $\left|S_{11}\right|$ in a wide band of frequencies.

mode of operation in a volumetric cylindrical resonator, in a coaxial slot resonator, and in a coaxial transmission line. There is a suppression of such signals.

At multiple frequencies, some signal penetration is observed. This is because of the incomplete suppression of oscillations due to the asymmetry of the design of the exciting coaxial slot resonator. It should be noted that other types of oscillations are excited in the cylindrical cavity resonator with other structures of electromagnetic fields at multiple frequencies. The relative dimensions of the aperture and radiating holes of the RA increase by several times, the complex radiation pattern, the amplitude, phase, and polarization characteristics of the antenna change. As a result, radio reception of unwanted out-of-band emissions becomes impossible. If the radio system is in transmission mode, it will create minimal interference with other systems.

Figure 3 shows the experimental frequency dependence of the modulus of the reflection coefficient in the operating frequency range. The passband of the RA at the level of VSWR 1.1 is equal to $1.8 \%$, and at the VSWR level of 1.45 is equal to $3 \%$. The squareness factor of the RA passband is equal to 2.67 .

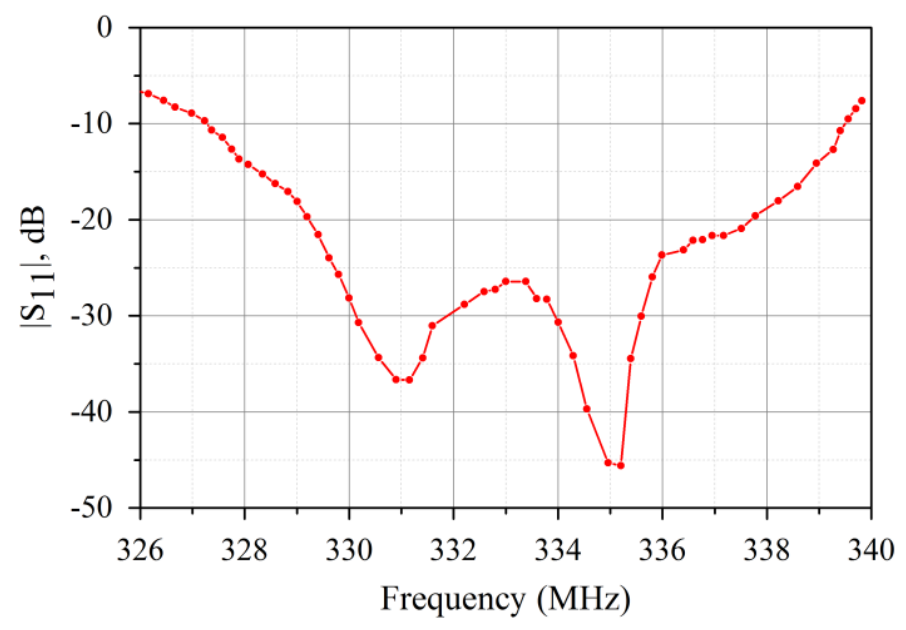

Fig. 3. Experimental frequency dependence of $\left|S_{11}\right|$ in the operating frequency range.

\subsection{Polarization characteristics of the resonant cavity antenna}

Table 1 presents the ratio of the main component amplitude of the electric field to the crosscomponent component in the plane of the magnetic field $\mathbf{H}$ in the working wavelength 
range. Table 2 presents the same characteristics in the plane of the electric field $\mathbf{E}$. $\lambda_{\min }=0,99 \lambda ; \lambda_{\max }=1,01 \lambda$.

Table 1. The ratio of the orthogonal components of the electric field vector $\mathbf{E}$ in the plane of the vector $\mathbf{H}$.

\begin{tabular}{|c|c|c|c|}
\hline \multirow{2}{*}{$\mathrm{e}^{\circ}$} & \multicolumn{3}{|c|}{ The ratio of orthogonal components, dB } \\
\cline { 2 - 4 } & $\lambda_{\max }$ & $\lambda$ & $\lambda_{\min }$ \\
\hline 0 & 60.30 & 60.80 & 59.11 \\
\hline 22 & 53.70 & 59.10 & 56.72 \\
\hline 45 & 44.67 & 51.30 & 53.74 \\
\hline 90 & 19.80 & 27.60 & 30.71 \\
\hline
\end{tabular}

Table 2. The ratio of the orthogonal components of the electric field vector $\mathbf{E}$ In the plane of the vector $\mathbf{E}$.

\begin{tabular}{|c|c|c|c|}
\hline \multirow{2}{*}{$\mathrm{Q}^{\circ}$} & \multicolumn{3}{|c|}{ The ratio of orthogonal components, dB } \\
\cline { 2 - 4 } & $\lambda_{\max }$ & $\lambda$ & $\lambda_{\min }$ \\
\hline 0 & 60.30 & 60.80 & 59.11 \\
\hline 19 & 58.90 & 65.87 & 71.40 \\
\hline 45 & 57.32 & 57.19 & 48.54 \\
\hline 90 & 56.78 & 55.91 & 55.33 \\
\hline
\end{tabular}

According to Table 1 and Table 2, the antenna has good polarization characteristics. Within the main lobe of the pattern, the ratio of the main component of the electric field to the cross-component component is approximately minus $53-71 \mathrm{~dB}$. Within the working half-space, no change in the type of polarization is observed. Changes in the ratio of orthogonal components and phase shifts are negligible.

\subsection{Directional properties of a resonant cavity antenna}

The amplitude pattern (Fig. 4) of the resonant cavity antenna in the plane of the magnetic field $\mathrm{H}$ has only one main lobe (without side lobes in the anterior half-space). The width of the beam at the half power level in the plane of the magnetic field vector is equal to 43.8 degrees. The width of the beam at the level of half power in the plane of the vector of electric field strength is equal to 38.2 degrees. The level of the side lobes in the range of meridional angles \pm 150 degrees: in the plane of the magnetic field $\mathbf{H}$ is equal to minus $45 \mathrm{~dB}$; in the plane of the electric field $\mathbf{E}$ is equal to minus $22.3 \mathrm{~dB}$. The coefficient of directional action of the RA is equal to $13.3 \mathrm{~dB}$.

The resonant cavity antenna forms a quasi-plane front of electromagnetic waves in the near and intermediate zones. Within the main lobe of the amplitude radiation pattern, the deviations of the phase radiation pattern from the plane do not exceed 5 degrees.

The directivity characteristics of the RA correspond to the limiting theoretical directivity characteristics for a given radiating aperture. This is because the RA works as a Fabry-Perot resonator. In contrast to the resonators of the quasi-optical and optical wavelength ranges, the RA operates in a single-mode and has aperture sizes commensurate with the operating wavelength.

The antenna is characterized by a low level of lateral radiation, a low level of cross polarization radiation, a high coefficient of aperture utilization, low intrinsic noise temperature, and a high spatiotemporal selectivity. 


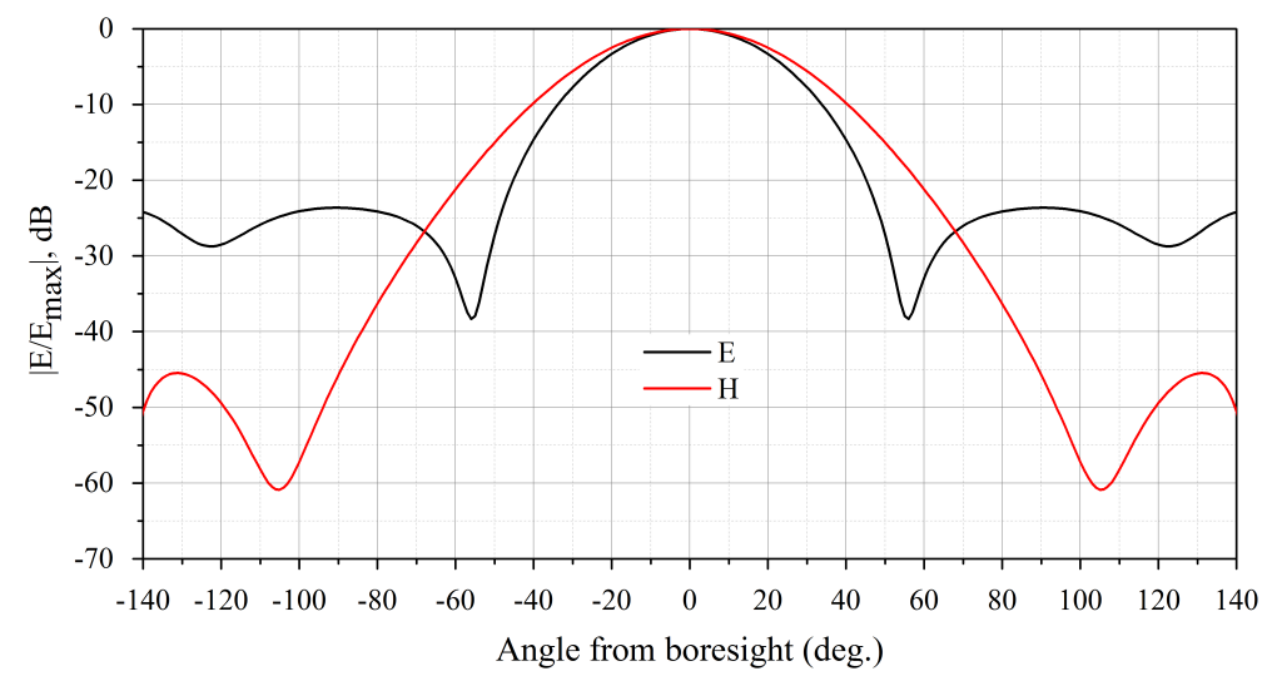

Fig. 4. Cavity antenna pattern.

\section{Conclusion}

The obtained results indicate that the resonant cavity antenna has unique selective characteristics. It has a high efficiency, a low level of side and rear radiation, a low level of cross-polarization radiation, low noise temperature and high frequency selectivity.

The simple design of the RA combined with high spatial and temporal selectivity ensure high stability and noise immunity of the radio engineering system, reduces the influence of external radiation, meteorological environmental factors and the underlying surface.

\section{References}

1. G. von Trentini, "Partially Reflecting Sheet Arrays," IRE Trans. Antennas Propag., vol. 4, no. 4, pp. 666-671, October (1956)

2. A. Kumar, H. D. Hristov, Microwave Cavity Antennas, Artech House (1989)

3. F. Meng, and S. K. Sharma, "A Dual-Band High Gain Resonant Cavity Antenna with A Single Layer Superstrate," IEEE Trans. Antennas Propag., vol. 63, issue 5, pp. 23202325, DOI: 10.1109/TAP.2015.2405082 (May 2015)

4. K. Konstantinidis, A.P. Feresidis, and P.S. Hall, "Multilayer Partially Reflective Surfaces for Broadband Fabry-Perot Cavity Antennas," IEEE Trans. Antennas Propag., vol. 62, no. 7, pp. 3474-3481, July (2014)

5. X. Chen, Z. Luo, Z. Zheng, P. Feng, and K. Huang, "Effective Reflective Characteristics of Superstrates and Their Effects on the Resonant Cavity Antenna," IEEE Trans. Antennas Propag., vol. 63, no. 4, pp. 1572-1580, DOI: 10.1109/TAP.2015.2399508 (April 2015)

6. Muhannad A. Al-Tarifi, Dimitris E. Anagnostou, Anthony K. Amert, and Keith W. Whites, "Bandwidth Enhancement of the Resonant Cavity Antenna by Using Two Dielectric Superstrates,” IEEE Trans. Antennas Propag., vol. 61, no. 4, pp. 18981908, April (2013)

7. Qiang Chen, Xing Chen, and Ke Xu. 3-D Printed Fabry-Perot Resonator Antenna with Paraboloid-Shape Superstrate for Wide Gain Bandwidth. Applied Sciences, 7, 1134, DOI:10.3390/app7111134 (2017) 
8. Lu-Yang Ji, Pei-Yuan Qin, and Y. Jay Guo, "Wideband Fabry-Perot Cavity Antenna With a Shaped Ground Plane," IEEE Access, vol. 6, pp. 2291-2297, , DOI: 10.1109/ACCESS.2017.2782749 (December 2017)

9. R.M. Hashmi and K.P. Esselle, “A Class of Extremely Wideband Resonant Cavity Antennas with Large Directivity-Bandwidth Products, ” IEEE Trans. Antennas Propag., vol. 64, no. 2, pp. 830-835, DOI: 10.1109/TAP.2015.2511801 (Feb. 2016)

10. A. Brizzi, A. Pellegrini, and Y. Hao, "Design of a Cylindrical Resonant Cavity Antenna for BAN Applications at $V$ Band, ” IEEE International Workshop on Antenna Technology (iWAT), pp. 152-155, DOI: 10.1109/IWAT.2012.6178421 (2012)

11. N.I. Voytovich, V.A. Bukharin, A.V. Ershov, N.N. Repin, Flat cavity antenna, Proc. of 6th European Conference on Antennas and Propagation (EuCAP 2012), pp. 29002903, DOI: 10.1109/EuCAP.2012.6205876 (2012)

12. V.A. Bukharin, N.I. Voytovich, Space-Time Selectivity of a Flat Cavity Antenna, $29^{\text {th }}$ International Crimean Conference "Microwave \& Telecommunication Technology" (CriMiCo'2019), Sevastopol, Russia (2019)

13. V.A. Bukharin, N.I. Voytovich, A.V. Ershov, The Flat Cavity Antenna with the Circular Aperture, Proc. of the International Scientific-Practical Conference "SVYAZPROM 2007" within the limits of $4^{\text {th }}$ Euro-Asian forum "SVYAZPROMEKSPO 2007”, Russia, Yekaterinburg, "Real-Media Co. Limited”, pp. 288-290 (2007)

14. V.A. Bukharin, N.I. Voytovich, Flat C avity Antenna phase characteristics, $27^{\text {th }}$ International Crimean Conference "Microwave \& Telecommunication Technology" (CriMiCo'2017), Sevastopol, Russia, November 5-11, vol. 3, pp. 710-716 (2017)

15. T. Weiland, A Discretization Method for the Solution of Maxwell's Equations for SixComponent Fields, Electronics and Communication, (AEÜ), vol. 31, pp. 116-120 (1977) 